\title{
Personalidades vocacionais e generatividade na vida adulta
}

\author{
Mauro de Oliveira Magalhães \\ William B. Gomes \\ Universidade Federal do Rio Grande do Sul
}

\begin{abstract}
RESUMO
O interesse vocacional é uma expressão da personalidade que pode revelar maior ou menor predisposição para a criatividade e para o envolvimento na comunidade. Generatividade refere-se à preocupação com o bem-estar das próximas gerações. A pesquisa investigou as relações entre tipos de interesse vocacional e generatividade em dois estágios da vida adulta. Os sujeitos foram 415 homens e 321 mulheres, divididos em adultez inicial e adultez média, com idades entre 25 e 40, e 45 e 63 anos, respectivamente. Os interesses vocacionais foram categorizados de acordo com a tipologia de Holland. A generatividade foi medida pela Loyola Generativity Scale. Os elevados escores de generatividade de tipos empreendedores e sociais corroboram a proposição de que o traço de extroversão é fundamental para caracterizar o funcionamento generativo como uma orientação vigorosa e positiva do indivíduo para o mundo exterior. Já a combinação entre interesses realistas e convencionais caracteriza pessoas com limitações em criatividade e competência social, resultando em menor generatividade.
\end{abstract}

Palavras-chave: generatividade; interesses vocacionais; desenvolvimento adulto.

\begin{abstract}
Vocational personalities and generativity in adult life

Vocational interests are an expression of personality that can reveal more or less predisposition to creativity and pro-social behaviors Generativity is the concern with the welfare of the next generations. This research investigated the relations between vocational interests and generativity in two stages of adult life. The subjects were 415 men and 321 women, divided in early and middle adulthood, with ages ranging between 25 and 45 years, and 45 and 63 years, respectively. The vocational interests were classified through Holland typology. Generativity was measured by Loyola Generativity Scale. The higher generativity scores of social and enterprising types confirm the proposition that extroversion is fundamental to characterize generativity functioning as a strong and positive orientation to the external world. The combination between conventional and realistic interests characterizes people with limitations in creativity and social competence, resulting in lower generativity.
\end{abstract}

Keywords: generativity; vocational interests; adult development.

\section{INTRODUÇÃO}

Em meados do século passado, Erik Erikson dedicou-se ao tema da preocupação humana com o futuro e bem-estar das próximas gerações, a qual denominou de generatividade (Erikson \& Erikson, 1998). De acordo com o autor, as questões da generatividade emergem mais intensamente nos anos da adultez média. Neste período, a necessidade de cuidar expandese a partir do núcleo das relações de intimidade e caminha no sentido de uma preocupação com o futuro da espécie humana. Esta preocupação pode ser expressa na criação e produção de bens culturais duradouros ou no cuidado de outras pessoas. Neste sentido, na fase média da vida, o indivíduo atinge o topo das realizações profissionais em termos de criatividade e produtividade, freqüentemente assumindo papéis de liderança e destaque na sua área profissional e também na comunidade (Smolak, 1992). Além do 
trabalho, a educação dos filhos é a expressão mais comum de generatividade. Por outro lado, Erikson esclareceu que ter filhos não é uma garantia de que se está sendo generativo, pois é necessário um interesse genuinamente altruísta no cuidado com as próximas gerações, sejam estas representadas pelos próprios filhos ou pelos filhos de outrem (Erikson \& Erikson, 1998).

Segundo McAdams e de St. Aubin (1998), o significado da generatividade revela dois aspectos interligados: (a) a criação, produção ou geração de algo; e (b) a doação deste algo como uma dádiva pessoal para a posteridade. Ora, a criação perde o sentido generativo se o que foi criado não é ofertado à comunidade. $\mathrm{E}$ a doação de algo é vazia quando o indivíduo não identifica a sua singularidade naquilo que doa. Os autores elaboraram a Escala de Preocupações Generativas ou Loyola Generativity Scale a fim de mensurar a generatividade (McAdams \& de St. Aubin, 1998).

As pesquisas têm apontado relações entre personalidade e critérios de maturidade psicossocial, entre estes a generatividade. Bradley e Marcia (1998) e Helson e Strivastava (2001) sugeriram diferentes estilos de generatividade e descreveram padrões de maturidade na vida adulta. Estas pesquisas serão apresentadas no tópico a seguir.

\section{Diferenças individuais e generatividade}

Bradley e Marcia (1998) propuseram um modelo de 5 categorias de estados de generatividade, com base em dois critérios: envolvimento e inclusividade. Envolvimento significa a capacidade de comprometerse com projetos de desenvolvimento pessoal e/ou da coletividade. E a inclusividade refere-se a quem vai ser incluído ou excluído das preocupações generativas. Baixos níveis de inclusividade refletem a característica de rejeição, tanto em termos de uma autorejeição, quanto da indisposição para incluir determinadas pessoas ou grupos em suas preocupações generativas. A ausência de inclusividade está relacionada ao autoritarismo e a intolerância às diferenças culturais.

As categorias de estado generativo descritas por Bradley e Marcia (1998) foram as seguintes: generativo, convencional, agência e comunal. A categoria generativo caracteriza o resultado mais positivo, pois apresenta níveis elevados de envolvimento e inclusividade. Estas pessoas são altamente envolvidas no trabalho e preocupadas com questões sociais. São tolerantes às diferenças culturais e, portanto, suas preocupações sociais são mais abrangentes. Por outro lado, são capazes de encontrar o equilíbrio entre o cuidado de si mesmo e dos demais. A categoria con- vencional significa que o indivíduo tende a apresentar alto grau de envolvimento generativo, porém baixo grau de inclusividade para si mesmo e para os outros. Isto significa que está focado no atendimento das normas e demandas sociais de uma coletividade específica, mostrando dificuldade de ampliar seus interesses para incluir outros aspectos de si mesmo e outros grupos sociais. $\mathrm{O}$ estado convencional tem uma visão de mundo restrita, considera que a juventude precisa de uma orientação firme e tem dificuldade para aceitar valores e culturas diversas. $\mathrm{O}$ estado de agência significa alto envolvimento com interesses pessoais e exclusão das pessoas não envolvidas nestes projetos. $\mathrm{O}$ estilo comunal tem um alto investimento nos outros, deixando suas ambições e desejos pessoais num plano secundário. E, por fim, o tipo estagnado revela o pior resultado possível. Neste caso, as interações com o mundo são mínimas e a satisfação pessoal também.

Bradley e Marcia (1998) encontraram correlações entre a sua Medida de Estados Generativos (Generativity Status Measure - GSM) e outros instrumentos e critérios tais como a Loyola Generativity Scale (McAdams \& de St. Aubin, 1998) e o modelo dos cinco grandes fatores de personalidade, também denominado de Big Five (McCrae \& Costa, 2002). Este modelo propõe que a personalidade pode ser sumariada através de cinco dimensões amplas e ortogonais, rotuladas de neuroticismo, extroversão, abertura à experiência, socialização e escrupulosidade. Em linhas gerais, neuroticismo é a predisposição para experimentar afetos negativos. Extroversão significa espírito gregário e assertividade. $\mathrm{O}$ traço de abertura quer dizer uma disposição para novas experiências, sensibilidade estética e flexibilidade. A sociabilidade engloba aspectos de altruísmo, cuidado, confiança e cooperação. E por fim, escrupulosidade inclui persistência, disciplina, responsabilidade e necessidade de realização (McCrae \& Costa, 2002).

No estudo de Bradley e Marcia (1998), o estado generativo correlacionou positivamente com os fatores extroversão $(r=.24)$ e abertura à experiência $(\mathrm{r}=.46)$. $\mathrm{O}$ estado de agência correlacionou negativamente com sociabilidade (do original agreeableness) $(\mathrm{r}=-.23)$ e o estado comunal correlacionou positivamente $(\mathrm{r}=.28)$ com o mesmo fator. $\mathrm{O}$ estado convencional mostrou associação positiva com escrupulosidade $(\mathrm{r}=.23)$ e negativa com abertura $(\mathrm{r}=-.47)$. E o estado estagnado correlacionou negativamente com extroversão e escrupulosidade $(r=-.30)$. Os estados de agência e generativo obtiveram os escores mais elevados nas medidas de generatividade, e os estados convencional e estagnado obtiveram os mais baixos. $\mathrm{O}$ traço de abertura à experiência se mostrou positi- 
vamente associado com a dimensão de inclusividade e, portanto, discriminou os estados generativo e convencional. Nas pesquisas de Auge (1996) e de St. Aubin e McAdams (1995) a generatividade correlacionou positivamente com os traços de sociabilidade, extroversão e abertura à experiência, e negativamente com neuroticismo.

Helson e Strivastava (2001) propuseram uma tipologia de padrões de maturidade psicossocial na vida adulta. Esta tipologia está baseada na combinação de dois critérios de maturidade: domínio ambiental e crescimento pessoal. Domínio ambiental (DA) significa a capacidade de alcançar adequação no ambiente, fazendo uso das oportunidades disponíveis e lidando de modo competente com as demandas da vida prática. Crescimento pessoal (CP) significa a capacidade de aprendizagem e mudanças no âmbito intrapsíquico. Os autores acreditam que, no curso do desenvolvimento, a ênfase numa destas orientações tende a obstar a outra. Os indivíduos podem ser categorizados em quatro tipos: realizadores (DA e $\mathrm{CP}$ elevados), conservadores (DA elevado, CP baixo), investigadores (DA baixo, CP elevado) e debilitados (DA e CP baiXos). A pesquisa longitudinal de Helson e Strivastava (2001) comparou estes tipos em várias medidas de personalidade e maturidade psicossocial, entre estas a generatividade. Os conservadores apresentaram menor abertura à experiência, sabedoria e desenvolvimento do ego; por outro lado, revelaram maior competência social e escores elevados em autocontrole. Os investigadores mostraram maior abertura à experiência, sabedoria e desenvolvimento do ego; e os menores escores em autocontrole. A categoria dos realizadores se mostrou a mais competente e generativa, mas não se destacou em termos de sabedoria e desenvolvimento do ego. Os debilitados apresentaram o pior desempenho nestas variáveis. Os conservadores apresentaram mais evidências de que aceitaram a identidade que lhes foi atribuída, enquanto investigadores buscaram a resolução de conflitos de identidade. Os conservadores revelaram inibição de respostas emocionais tanto positivas quanto negativas, em contraste com a abertura para a vida emocional demonstrada pelos investigadores. Os autores argumentaram que alguns indivíduos restringem suas emoções para se conformarem a normas que consideram importantes e certas; e outros dão livre expressão a sentimentos e inclinações. Sendo assim, sugeriram que realizadores e conservadores investiram para realizar metas como promoções no trabalho e estabilidade no casamento, que são mais facilmente alcançadas pela aderência às normas sociais; enquanto investigadores buscaram desenfatizar estas normas e aprender a confiar na intuição em busca do crescimento pessoal.

\section{Personalidades vocacionais e generatividade}

As realizações profissionais têm grande importância para a generatividade, pois, de um modo geral, é através do trabalho que as pessoas encontram a oportunidade de apresentar uma contribuição pessoal e singular para a coletividade. Na concepção de McAdams e de St. Aubin (1998), o trabalho terá sentido generativo na medida em que resultar num legado a ser ofertado às próximas gerações. A construção deste legado implica criar algo original, identificado com o self, e orientado para o bem-estar da coletividade. Neste sentido, podese dizer que as atividades de trabalho que não propiciam o exercício da criatividade não são favoráveis à experiência da generatividade. $\mathrm{E}$, por outro lado, quando o trabalho é desprovido de conteúdo social, também se pode dizer que dificultará a experiência da generatividade. Enfim, será possível identificar contextos de trabalho mais ou menos favoráveis à generatividade? E qual será o papel das diferenças de personalidade neste processo? A teoria de Holland (1997) oferece uma tipologia de personalidades e ambientes vocacionais que pode ser útil na busca destas respostas.

Holland (1997) considera que a escolha de uma carreira reflete a personalidade do sujeito. Ele propõe que os membros de uma profissão têm personalidades similares e histórias similares de desenvolvimento pessoal. $\mathrm{O}$ autor postula que as pessoas e os ambientes de nossa cultura podem ser caracterizados de acordo com seis tipos pessoais e ambientais, a saber: realista, investigativo, artístico, social, empreendedor e convencional. As personalidades vocacionais são definidas pelo maior ou menor interesse que o sujeito possui por cada um dos ambientes ocupacionais. O interesse predominante caracteriza o tipo de personalidade. $\mathrm{O}$ tipo realista é avesso à interação social, preferindo manipular máquinas e lidar com problemas concretos. $\mathrm{O}$ tipo investigativo prefere atividades de reflexão e criatividade no plano das idéias. O tipo artístico está focado na criação de formas estéticas usando idéias e emoções. O tipo social está voltado para o cuidado de outras pessoas. $\mathrm{O}$ indivíduo empreendedor usa a persuasão para liderar grupos e iniciar empreendimentos. E o tipo convencional prefere situações estruturadas, estáveis e metódicas. Note-se que estas breves descrições apresentadas tratam de tipos extremos. Na verdade, cada indivíduo possui características de todos os seis tipos em maior ou menor grau, ainda que os atributos de um dado tipo possam predominar. Um escore é calculado para cada interesse vocacional, e o escore mais elevado define a classificação tipológica de um sujeito particular. 
As pesquisas geradas pelo modelo de Holland (1997) corroboraram as suas idéias e fazem-no o mais influente no campo da psicologia vocacional. Esta tipologia tem mostrado relações consistentes com traços de personalidade, notadamente com os cinco grandes fatores (Larson, Rotinghaus \& Borgen, 2002). E a generatividade também tem sido relacionada ao modelo dos cinco grandes fatores (eg., Auge, 1996).

As pesquisas encontraram relações entre os traços de abertura à experiência, extroversão, sociabilidade e preocupações generativas (Auge, 1996; Bradley \& Marcia, 1998; de St. Aubin \& McAdams, 1995). Ora, considerando a generatividade como o desejo de trazer contribuições únicas e criativas para a comunidade, isto implica a combinação de criatividade (abertura à experiência) e interesse social (extroversão/sociabilidade). Sendo assim, os tipos de personalidade vocacional associados à generatividade devem apresentar estas características. No modelo de Holland (1997), estes traços são encontrados nas personalidades social, empreendedora, artística e investigativa. $\mathrm{Na}$ pesquisa de Larson, Rotinghaus e Borgen (2002), $\mathrm{o}$ fator abertura foi associado a interesses artísticos e investigativos, o fator extroversão a interesses sociais e empreendedores, e o fator sociabilidade a interesses sociais. Por outro lado, as inclinações realista e convencional possuem características que não sugerem generatividade; pois, de acordo com Holland (1997), a primeira possui limitações em competências sociais (tendência à introversão) e a segunda mostra restrições em criatividade (baixos escores em abertura à experiência).

O envolvimento na comunidade é um indicativo de generatividade (McAdams \& de St. Aubin, 1998). Sendo assim, a generatividade requer habilidades interpessoais. Para Holland (1997), tipos convencionais e realistas estão entre os menos habilidosos socialmente. O tipo realista é o menos competente neste aspecto, pois considera estressante e rejeita tarefas que impliquem interação social, tais como ensinar, ajudar ou supervisionar pessoas. O tipo convencional carece de espontaneidade e abertura para interagir socialmente, e o faz de uma forma rígida e estereotipada.

$\mathrm{O}$ interesse vocacional convencional se mostrou correlacionado com o traço de escrupulosidade, indicando que tipos convencionais tendem a ser organizados, disciplinados, confiáveis, persistentes e competentes (Hogan \& Ones, 1997; Costa \& McRae, 1985). Por outro lado, a escrupulosidade foi associada à preferência pelas gratificações extrínsecas do trabalho, tais como salários e promoções (Amabile, Hill, Hennessey \& Tighe, 1994). E, no estudo de Boudreau, Boswell e Judge (2001), correlacionou negativamente com sucesso intrínseco na carreira (um construto relacionado à satisfação no emprego, na carreira e na vida). Portanto, a associação entre interesse convencional e escrupulosidade pode significar que tipos convencionais sejam excessivamente dedicados ao cumprimento de expectativas relacionadas ao contexto formal do trabalho, em detrimento de realizações intrapessoais. Além disto, considerando que tipos convencionais têm escores baixos em abertura à experiência e são pessoas restritas em seus interesses, podem ter dificuldades para encontrar atividades alternativas e gratificantes fora do contexto de trabalho. Este conjunto de tendências da personalidade vocacional convencional pode resultar em generatividade reduzida, considerando a falta de recompensas intrínsecas no trabalho e a relativa incapacidade de encontrar estas compensações em outros contextos.

Entre as categorias de estado generativo propostas por Bradley e Marcia (1998) (generativo, agêntico, comunal, convencional e estagnado), o estado convencional mostrou associação positiva com escrupulosidade e negativa com abertura, correspondendo às características da personalidade vocacional batizada com o mesmo nome por Holland (1997). Além disto, nos achados de Bradley e Marcia (1998), o estado convencional de generatividade caracterizou indivíduos de valores rígidos, que rejeitam a diversidade cultural e, portanto, restringem as suas preocupações generativas a grupos determinados. De acordo com os achados de Blake, Davison e Sackett (1997), estas características também correspondem ao interesse convencional de Holland (1997). No estudo de Blake e cols. (1997), o interesse convencional correlacionou com tendências à rigidez de valores, aversão à permissividade e pouca abertura para mudanças pessoais. Enfim, estes dados sugerem que a personalidade vocacional convencional, tal como o estado generativo convencional, apresente escores inferiores em generatividade em comparação com tipos vocacionais caracterizados pela abertura à experiência e tolerância à complexidade, quais sejam, os tipos artísticos e investigativos.

Na tipologia de maturidade psicossocial de Helson e Strivastava (2001), o contraste entre conservadores e investigadores sugere, de modo análogo, a contraposição entre tipos convencionais e investigativos na tipologia de interesses de Holland (1997). Assim como o tipo vocacional convencional, o padrão de maturidade conservador caracterizou-se por autocontrole, conformidade a normas sociais e baixos escores em abertura à experiência. De forma oposta, tipos investigadores (Helson \& Strivastava, 2001) e investigativos (Holland, 1997) se mostraram abertos à experiência, intuitivos, originais e voltados para a exploração 
intrapessoal. O padrão de maturidade conservador não foi associado com generatividade, sugerindo que o interesse convencional também possua limitações neste sentido.

Dollinger, Ross e Preston (2002) observaram que o interesse investigativo, associado com abertura à experiência, também está ligado ao construto individualidade, definido como a construção e apresentação do self de modo a ser diferente dos demais. Em contraste, a tendência à rigidez de tipos convencionais sugere um afastamento de reflexões sobre a sua identidade ou propósito de vida. Estes indivíduos tenderiam a assumir os estereótipos culturais sem questionamento e, deste modo, reduzem os seus potenciais criativos de oferecer novas e melhores alternativas de vida às próximas gerações.

As atividades profissionais preferidas pelo tipo convencional são metódicas, estruturadas e previsíveis, tais como tarefas de classificação, inspeção, contabilidade e conferência. Entre as profissões enquadradas nesta categoria estão os bancários, bibliotecários, contadores, analistas de crédito, arquivistas, entre outros. Algumas destas atividades têm sido alvo de pesquisas sobre estresse e sofrimento no trabalho (Codo, Sampaio, Hitomi, \& Bauer, 1993).

Fernandes (1999) utilizou uma medida de propósito vital, o PIL-Test (Purpose in life-test) (Crumbaugh, 1968), para comparar uma amostra de trabalhadores bancários com profissionais de outras áreas. O PILTest é uma medida indireta da depressão relacionada ao vazio existencial (Murray, 1987). Os bancários se mostraram mais propensos a este vazio existencial. $\mathrm{Na}$ pesquisa de Grossbaum e Bates (2002), a generatividade correlacionou com propósito vital $(\mathrm{r}=.52)$. Estes estudos sugerem que trabalhadores bancários apresentam escores inferiores em generatividade em comparação a outros profissionais.

Codo, Sampaio, Hitomi, e Bauer (1993) descreveram o mal-estar do trabalho vazio em bancários. De acordo com os autores, as características e a organização da atividade do bancário impede que o indivíduo se identifique com o produto do seu trabalho, resultando num processo de alienação. Porém, esta pesquisa e a de Fernandes (1999) não verificaram se os sujeitos investigados estavam vocacionalmente interessados na sua tarefa profissional, e atribuíram os seus resultados às características do contexto do trabalho bancário.

Por outro lado, numa perspectiva disposicional, Graham (1999) utilizou o MMPI e descreveu os bancários como egocêntricos, em conflito com necessidades de dependência, carentes de atenção e afeto, e socialmente superficiais. E, para Holland (1997), o indivíduo do tipo convencional, que revela interesse pela ocupação de bancário, é pouco assertivo, tende à obediência, tem baixa auto-estima e quer alcançar status sendo um bom subordinado. Estas descrições sugerem predisposições desfavoráveis à criatividade e a uma identificação com os aspectos intrínsecos da tarefa profissional.

Além dos fatores contextuais e disposicionais citados, a insatisfação e o sofrimento no trabalho também podem decorrer da incompatibilidade entre personalidade e tarefa (Holland, 1997). Portanto, torna-se relevante verificar em que medida a síndrome do trabalho vazio, associada às ocupações preferidas pelo tipo convencional, está relacionada a fatores contextuais, disposicionais, ou à incompatibilidade entre estes.

Numa abordagem disposicional, esta pesquisa investigou as relações entre personalidades vocacionais e generatividade. Estas relações foram verificadas em dois estágios da vida adulta: adultez inicial e adultez média (Levinson, 1985). Hipotetizou-se que personalidades convencionais e realistas mostrem escores inferiores em generatividade, comparados com sociais, empreendedores, investigativos e artísticos; e que os sujeitos na meia-idade apresentem escores mais elevados de generatividade.

\section{MÉTODO}

\section{Sujeitos}

Participaram do estudo 736 profissionais. Entre eles, 371 (198 homens e 173 mulheres) situados no estágio da adultez inicial, com idades entre 25 e 40 anos $(M=33,2)$ e, no mínimo, 5 anos de atividade formal de trabalho $(M=11,2)$. E 362 (217 homens e 145 mulheres) situados na adultez média, com idades entre 44 e 60 anos $(M=49)$ e com mais de 15 anos de carreira $(\mathrm{M}=22,7)$. Na adultez inicial, $16 \%$ possuíam ensino médio completo e formação técnica, $34 \%$ estavam cursando ensino superior, $31 \%$ possuíam graduação superior e $18 \%$ eram pós-graduados. E na adultez média, $16 \%$ possuíam ensino médio completo e formação técnica, $26 \%$ estavam cursando ensino superior, $38 \%$ possuíam graduação superior e $19 \%$ eram pósgraduados.

\section{Instrumentos e Procedimentos}

Os sujeitos responderam a medidas de generatividade e interesses vocacionais. A generatividade foi mensurada pela Loyola Generativity Scale (McAdams 
$\&$ de St. Aubin, 1998). Este instrumento é composto de 20 itens com chave de respostas no formato Likert de 5 pontos. A versão brasileira obteve índice de consistência interna (alpha de Cronbach) de .83. Os interesses vocacionais foram mensurados pela escala de atividades do Self Directed Search (SDS - Holland, 1997), que lista seis conjuntos de 11 atividades, correspondentes aos seis tipos de interesse vocacional (R, I, A, S, E, C). Os sujeitos informaram a preferência por estas atividades numa escala Likert de 5 pontos. Esta escala mostrou qualidades psicométricas satisfatórias em amostras brasileiras (Balbinotti, Magalhães, Callegari \& Fonini, 2004). Os sujeitos responderam aos instrumentos no seu local de trabalho ou estudo. Após esclarecimento sobre os objetivos da pesquisa, assinaram o termo de consentimento livre e esclarecido.

\section{Delineamento}

Foram analisadas as diferenças de generatividade relacionadas ao estágio de vida e aos tipos de interesse vocacional. Também foi utilizado um delineamento fatorial 2X6 (2 níveis de estágio de vida e seis tipos de orientação de personalidade vocacional) tendo como variável dependente a generatividade. Este delineamento se repetiu em duas análises. Na primeira, consideraram-se os seis agrupamentos de personalidade vocacional de acordo com o interesse mais elevado
(R, I, A, S, E, C). Na segunda, os sujeitos foram agrupados de acordo com os dois interesses adjacentes e com maiores escores (RI, RC, IA, ES, EC, AS). Os perfis foram definidos a partir dos escores brutos, considerando as diferenças intrasujeito, de acordo com as recomendações de Holland (1997).

\section{RESULTADOS}

Os sujeitos situados no estágio da adultez média obtiveram escores mais elevados de generatividade $(\mathrm{M}=74,97 ; \mathrm{DP}=10,80)$ do que os sujeitos situados na adultez inicial $(\mathrm{M}=72,78 ; \mathrm{DP}=11,89), \mathrm{F}(1,721)$ $=5.30, \mathrm{p}<.05$.

A análise de variância indicou diferenças de generatividade entre os tipos vocacionais definidos pelo interesse predominante, $\mathrm{F}(5,655)=2.72, \mathrm{p}<.05$. A tabela 1 apresenta a estatística descritiva dos escores de generatividade em cada tipo vocacional. Os testes post hoc (Tukey) revelaram que os tipos sociais, empreendedores e artísticos apresentaram escores mais elevados de generatividade em comparação com os convencionais. Não houve interação entre tipo vocacional e estágio de vida para variação em generatividade no delineamento fatorial 2X6 (2 níveis de estágio de vida e seis tipos de orientação de personalidade vocacional - R, I, A, S, E, C).

Tabela 1. Médias e desvios-padrão de generatividade e valores de $\mathrm{N}$ em cada categoria vocacional definida pelo interesse predominante

\begin{tabular}{lccc}
\hline \multicolumn{1}{c}{ Tipos Vocacionais } & $\mathrm{N}$ & Média & Desvio-padrão \\
\hline Realista & 64 & 72.39 & 14.04 \\
Investigativo & 57 & 72.47 & 11.51 \\
Artístico & 94 & 74.77 & 10.65 \\
Social & 153 & 75.37 & 12.89 \\
Empreendedor & 246 & 74.87 & 10.79 \\
Convencional & 47 & 68.79 & 12.30 \\
\hline Total & 661 & 73.79 & 11.87 \\
\hline
\end{tabular}

A tabela 2 mostra os valores de $\mathrm{N}$, médias e desvios-padrão de generatividade para os tipos vocacionais definidos a partir da combinação dos dois interesses predominantes (RI, RC, IA, ES, EC, AS). A análise de variância indicou diferenças significativas de generatividade entre estes tipos vocacionais, $\mathrm{F}(5,668)=$ $5.06, \mathrm{p}<.01$. Os testes post hoc (Tukey) revelaram que as combinações de interesses empreendedores e sociais (ES) e sociais e artísticos (AS) obtiveram esco- res significativamente mais elevados do que a combinação de interesses realistas e convencionais (RC) e empreendedores e convencionais (EC). Não houve interação entre tipos vocacionais e estágio de vida para variação em generatividade no delineamento fatorial 2X6 (2 níveis de estágio de vida e seis tipos de orientação de personalidade vocacional - RI, RC, IA, $\mathrm{ES}, \mathrm{EC}, \mathrm{AS}$ ). 
Tabela 2. Médias e desvios-padrão de generatividade e valores de $\mathrm{N}$ em cada categoria vocacional definida pelos dois interesses predominantes

\begin{tabular}{cccc}
\hline Tipos vocacionais & $\mathrm{N}$ & Média & Desvio-padrão \\
\hline RI & 81 & 70,60 & 12,96 \\
RC & 25 & 72,68 & 10,64 \\
IA & 46 & 71,61 & 12,60 \\
EC & 125 & 71,20 & 11,11 \\
ES & 261 & 76,06 & 11,56 \\
SA & 136 & 74,66 & 11,53 \\
\hline Total & 674 & 73,79 & 11,86 \\
\hline
\end{tabular}

\section{DISCUSSÃO E CONCLUSÕES}

De acordo com as expectativas teóricas, os escores de generatividade foram superiores no estágio da adultez média (Erikson \& Erikson, 1998). Nesta etapa da vida, geralmente atinge-se o topo das realizações profissionais em produtividade, status e criatividade; e é mais freqüente o papel de tutor das próximas gerações (Smolak, 1992).

Os escores inferiores de generatividade do tipo convencional em comparação com tipos empreendedores, sociais e artísticos corresponderam às expectativas teóricas. A diferença encontrada entre os tipos ES e SA e os tipos RC confirmaram expectativas relacionadas a combinação entre tipos realistas e convencionais. A concepção da generatividade como a articulação de motivações comunais e de agência (McAdams \& de St. Aubin, 1998), ou a combinação de comportamento pró-social e criatividade/produtividade (Erikson \& Erikson, 1998), caracteriza, por ângulos diversos, os tipos sociais (motivação comunal, traço de sociabilidade), empreendedores (agência, traço de extroversão), e artísticos e investigativos (traço de abertura à experiência e criatividade). Portanto, as limitações em competência social do tipo realista e as limitações em criatividade do tipo convencional revelaram ter um impacto negativo na generatividade destes sujeitos. Embora o escore predominante R não tenha se revelado menos generativo do que os demais, a associação RC obteve os escores mais baixos. A combinação entre interesses realistas e convencionais caracteriza pessoas com limitações em criatividade e competência social, resultando em menor generatividade.

Os elevados escores de generatividade de tipos empreendedores e sociais corroboram a proposição de Bradley e Marcia (1998) de que o traço de extroversão é fundamental para caracterizar o funcionamento generativo como uma orientação vigorosa e positiva do indivíduo para o mundo exterior. A combinação de interesses empreendedores e sociais (ES), que apresentam correlações positivas com este traço de perso- nalidade (Larson, Rotinghaus \& Borgen, 2002), parece resultar numa orientação de personalidade altamente favorável à generatividade.

No estudo de Bradley e Marcia (1998), a categoria de estado generativo denominada convencional também mostrou escores inferiores de generatividade, além de outras características similares à personalidade convencional descrita por Holland (1997). A tendência conservadora e de rigidez moral relacionada ao interesse convencional (Blake \& cols., 1997) é comum à carência de inclusividade do estado generativo convencional (Bradley \& Marcia, 1998), e pode ser interpretada como a ausência do que Erikson (Erikson \& Erikson, 1998) referiu como uma crença básica e geral na validade e bondade da espécie humana (Erikson \& Erikson, 1998). Indivíduos que falham em adquirir e/ou fortalecer esta crença provavelmente restringem o âmbito das suas preocupações generativas. Sendo assim, os resultados deste estudo reforçam a similaridade entre estas categorias de indivíduos, e sugerem que o interesse convencional esteja relacionado a um padrão característico de desenvolvimento psicossocial na vida adulta.

Nesta perspectiva de entendimento, o interesse convencional também mostrou similaridade à descrição feita por Helson e Srivastava (2001) do padrão de maturidade psicossocial conservador. Os indivíduos conservadores, caracterizados por baixos escores em abertura à experiência, revelaram uma tendência a restringir emoções e indagações existenciais a fim de atender expectativas sociais de estabilidade e competência no trabalho e na vida pessoal. Se, por um lado, esta postura resultou em elevada competência social, trouxe efeitos negativos em termos de sabedoria e desenvolvimento do ego.

De acordo com Levinson (1985), uma estrutura de vida pode ser avaliada sob dois aspectos: viabilidade e adequação. O primeiro é a sua capacidade funcional objetiva de satisfazer necessidades na relação do self com o meio externo. $\mathrm{O}$ segundo significa o quanto permite a expressão livre do self. As características do 
interesse convencional Holland (1997), do estado generativo convencional (Bradley \& Marcia, 1998) e do padrão de maturidade conservador (Helson \& Srivastava, 2001) sugerem a capacidade de construir uma estrutura de vida viável e funcional na relação com o meio externo ao self. Por outro lado, parecem obstar a construção de canais adequados para a expressão autêntica e singular da individualidade. Especula-se que tipos convencionais tendem a apresentar uma vida organizada e materialmente confortável, porém carente de engajamento ou propósito; ou seja, com um alto grau de viabilidade, mas pouca adequação às aspirações do self. Neste contexto, as atividades de trabalho cumpririam demandas estritas de subsistência, segurança e inserção social; além de proverem um sentido de utilidade e produtividade. Porém, teriam menor importância como fonte de identidade e significado vital. Em contraste, outros tipos vocacionais, caracterizados por maior abertura à experiência (investigativos e artísticos) e orientação pró-social (sociais e empreendedores), estariam mais dispostos a buscar e encontrar gratificações intrínsecas na tarefa profissional.

No presente estudo, os sujeitos classificados como tipos convencionais responderam a um inventário de preferências vocacionais para serem situados nesta categoria. Portanto, sugere-se que a carência de preocupações generativas neste grupo de profissionais também está relacionada a fatores disposicionais e não somente aos aspectos contextuais citados pela literatura sobre o sofrimento no trabalho (Codo, Sampaio \& Hitomi, 1993).

Esta pesquisa confirmou a hipótese de que variações em generatividade estão associadas a tipos de interesse vocacional e corroborou a importância de fatores de personalidade, ou disposicionais, para o entendimento do desenvolvimento na vida adulta. Os escores inferiores de generatividade de tipos convencionais reforçaram a correspondência teórica e empírica com padrões de desenvolvimento e maturidade psicossocial anteriormente descritos, a saber: padrão de desenvolvimento conservador (Helson \& Srivastava, 2001) e estado de generatividade convencional (Bradley \& Marcia, 1998). Portanto, os resultados recomendam novos estudos que explorem as relações entre estilos de maturidade psicossocial e interesses vocacionais.

\section{REFERÊNCIAS}

Amabile, T. M., Hill, K. G., Hennessey, B. A. \& Tighe, F. M. (1994) The Work Preference Inventory: assessing intrinsic and extrinsic motivational orientations. Journal of Personality and Social Psychology, 66, 950-967.
Auge, S. (1996). The Big Five as they relate to generativity. Tese de Doutorado não publicada, Northwestern University, Evanston, IL.

Balbinotti. M, Magalhães, M., Callegari, S. \& Fonini, C. R. (2004). Estudo fatorial exploratório da versão brasileira do inventário de preferências profissionais. Em Sociedade Brasileira de Psicologia (Org.), Resumos da XXXIV Reunião Anual de Psicologia [CD-ROM]. Ribeirão Preto, SP: SBP.

Blake, R. J., Davison. M. \& Sackett, S. A. (1997). Interest profile parameters derived from multidimensional scaling. Em S. A. Sackett (Chair), Interpreting Vocational Interest Patterns: Research, Innovations, and Emerging Directions. Simpósio realizado no encontro anual da American Psychological Association, Chicago, Illinois.

Boudreau, J. W., Boswell, W. R. \& Judge, T. A. (2001). Effects of personality on executive career success in the Unites States and Europe. Journal of Vocational Behavior, 58(1), 53-81.

Bradley, C. J. \& Marcia, J. E. (1998). Generativity-stagnation: a five-category model. Journal of Personality, 66(1), 39-64.

Codo, W., Sampaio, J. J. C. \& Hitomi, A. H. (1993). Indivíduo, trabalho e sofrimento: uma abordagem interdisciplinar. Petrópolis: Vozes.

Codo, W., Sampaio, J. J. C., Hitomi, A. H. \& Bauer, M. (1993). O mal-estar do trabalho vazio em bancários. Jornal Brasileiro de Psiquiatria, 42(1), 23-28.

Costa, P. T., Jr. \& McCrae, R. R. (1985). The NEO Personality Inventory Manual. Odessa, FL: Psychological Assessment Resources.

Crumbaugh, J. C. (1968). Cross validation of the Purpose in Life test based on Frankl's concepts. Journal of Individual Psychology, 24, 74-81.

de St. Aubin, E. \& McAdams, D. P. (1995). The relations of generative concern and generative action to personality traits, satisfaction/happiness with life, and ego development. Journal of Adult Development, 2, 99-112.

Dollinger, S. J., Ross, V. \& Preston, L. A. (2002). Intellect and individuality. Creativity Research Journal, 14(2), 213-226.

Erikson, E. \& Erikson, J. M. (1998). Life cycle completed: Extended version. New York: Norton.

Fernandes, F. S. D. (1999). Motivação vital em bancários: um estudo comparativo. Monografia de conclusão de curso de graduação em psicologia. Canoas: Universidade Luterana do Brasil.

Graham, J. R. (1999). MMPI-2: Assessing Personality and Psychopathology. London: Oxford University Press.

Grossbaum, M. F., Bates, G. W. (2002). Correlates of psychological well-being at midlife: the role of generativity and agency and communion, and narrative themes. International Journal of Behavioral Development, 26 (2), 120-128.

Helson, R. \& Srivastava, S. (2001). Three paths of adult development: conservers, seekers and achievers. Journal of Personality and Social Psychology, 80(6), 995-1010.

Hogan, J. \& Ones, D. S. (1997). Conscientiousness and integrity at work. Em R. Hogan, J. Johnson \& S. Briggs (Orgs.), Handbook of personality psychology (849-870). San Diego: Academic Press. 
Holland, J. L. (1997). Making vocational choices: A theory of vocational personalities and work environments. Odessa, FL. PAR.

Larson, L. M., Rottinghaus, P. J. \& Borgen, F. H. (2002). Metaanalyses of Big Six Interests and Big Five Personality Factors. Journal of Vocational Behavior, 61(2), 217-239.

Levinson, D. (1985). The seasons of a man's life. New York: Alfred Knopf.

McAdams, D. P. \& de St. Aubin, E. (1998). Generativity and adult development. Washington, DC: APA.

McCrae, R. R. \& Costa, P. T. (2002) Personality in adulthood: a five-factor theory perspective. Nova York: Guilford.
Murray, D.J. (1987). Assessing logotherapeutic constructs: conceptual and psychometric status of the purpose in life and seeking of Noetic Goals tests. Journal of Clinical Psychology, 7(1) 201-202.

Smolak, L. (1992). Adult development. Englewood Cliffs: Prentice Hall.

Recebido: 06/08/2005

Revisado: $23 / 10 / 2005$

Aceito: $23 / 12 / 2005$

\section{Sobre os autores:}

Mauro de Oliveira Magalhães: Doutor em Psicologia pela Universidade Federal do Rio Grande do Sul (UFRGS), professor da Universidade Luterana do Brasil (Ulbra - Canoas/RS), e editor da revista Aletheia.

William B. Gomes: Professor do Programa de Pós-Graduação em Psicologia da Universidade Federal do Rio Grande do Sul, Bolsista Produtividade CNPq.

Endereço para correspondência: Instituto de Psicologia - UFRGS - Rua Ramiro Barcelos 2600, s/113 - 90035-005 Porto Alegre - RS (gomesw@ufrgs.br). 